\title{
A reaction to mitochondria in action
}

\author{
Mark P Mattson $^{1}$ \\ ${ }^{1}$ Laboratory of Neurosciences, National Institute on Aging Intramural Research Program, Baltimore, MD 21224 \\ Cell Research (2011) 21:1279-1282. doi:10.1038/cr.2011.87; published online 24 May 2011
}

In most cells and under most conditions, mitochondria produce the majority of the readily available energy (in the form of ATP) to sustain a myriad of biochemical processes necessary for the survival and proper function of the cell. Excitable cells, such as neurons and muscle cells, require very high amounts of ATP to maintain and restore the ion gradients across membranes as they experience frequent bouts of depolarization-induced $\mathrm{Na}^{+}$and $\mathrm{Ca}^{2+}$ influx [1]. Oxidative phosphorylation, the chemical process by which a highenergy phosphate bond is added to ADP to produce ATP, is mediated by electron transport chain (ETC) protein complex enzymes associated with the mitochondrial inner membrane (Figure 1). During the electron transport process superoxide anion radical (SO) is produced. Mitochondrial manganese superoxide dismutase (SOD) converts $\mathrm{SO}$ to the more stable and membrane permeable hydrogen peroxide, with the latter being converted to water via the actions of catalase and glutathione peroxidases. However, SO production can also lead to the generation of several highly destructive free radicals including hydroxyl radical and peroxynitrite [2]. Excessive production and/or reduced detoxification of $\mathrm{SO}$ is implicated in the pathogenesis of a range of diseases including cardiovascular disease [3],

Correspondence: Mark P Mattson

E-mail: MattsonM@grc.nia.nih.gov diabetes [4] and neurodegenerative disorders [5]. On the other hand, SO may serve as a signaling molecule that regulates cellular energy metabolism, growth and survival [6].

SO production occurs primarily at mitochondrial complexes 1 and 3. Because of the cascading nature of the ETC assembly, it is generally believed that SO production is directly proportional to ATP production, such that both SO and ATP production are regulated (under physiological conditions) mainly by $\mathrm{O}_{2}$ and energy substrate (e.g., glucose) availability. However, in pathological settings an aberrant sustained increase in $\mathrm{SO}$ production and a reduction in ATP production may occur as the result of impairment of complex 1, a scenario believed to happen in the dopamineproducing neurons that degenerate in Parkinson's disease [7]. But recent findings, bolstered by technological advances, challenge the concept that $\mathrm{SO}$ is normally produced in a graded manner proportional to ETC activity. In particular, the development of a genetically-engineered fluorescent SO probe provides a tool for observing SO production in individual mitochondria of living cells in real time [8]. Circular permuted yellow fluorescent protein targeted to mitochondria (mt-cpYFP) was shown to increase its fluorescence emission in response to $\mathrm{SO}$, but not other reactive oxygen species or ATP. When imaged in the mitochondria of cardiac myocytes and neurons, the cpYFP signal exhibited spontaneous 'flashes'; the frequency of the SO flashes was increased in response to hyperoxia, and the flashes were abolished by inhibition of the ETC, by SOD mimetics and by inhibition of mitochondrial membrane permeability transition pores (mPTP) (Figure 1). The latter discovery raised many new questions, including whether such mitochondrial SO flashes occur in vivo and, if so, what factors influence their occurrence.

Recently, Fang et al. [9] provide an important advance towards understanding the behaviors and functions of mitochondria in vivo. Previous attempts to study mitochondrial SO production and $\mathrm{mPTP}$ opening in vivo had involved measurements made using isolated mitochondria, or relied on indirect indicators of SO levels (hydroethidine fluorescence) and mPTP opening (cytochrome c release into the cytosol), and did not reveal the existence of mitochondrial SO flashes. The authors generated transgenic mice that express mt-cpYFP in all of their cells, and found that they could image mitochondria in skeletal muscle cells and axons of peripheral neurons by confocal microscopy in anesthetized mice. Mitochondria in skeletal muscle cells exhibited spontaneous SO flashes that occurred throughout the mitochondrial network of individual cells. SO flashes occurred asynchronously in individual mitochondria distributed along axons of the sciatic nerve and, interestingly, SO flashes occurred only in mitochondria that were stationary and not in mitochondria that were being 


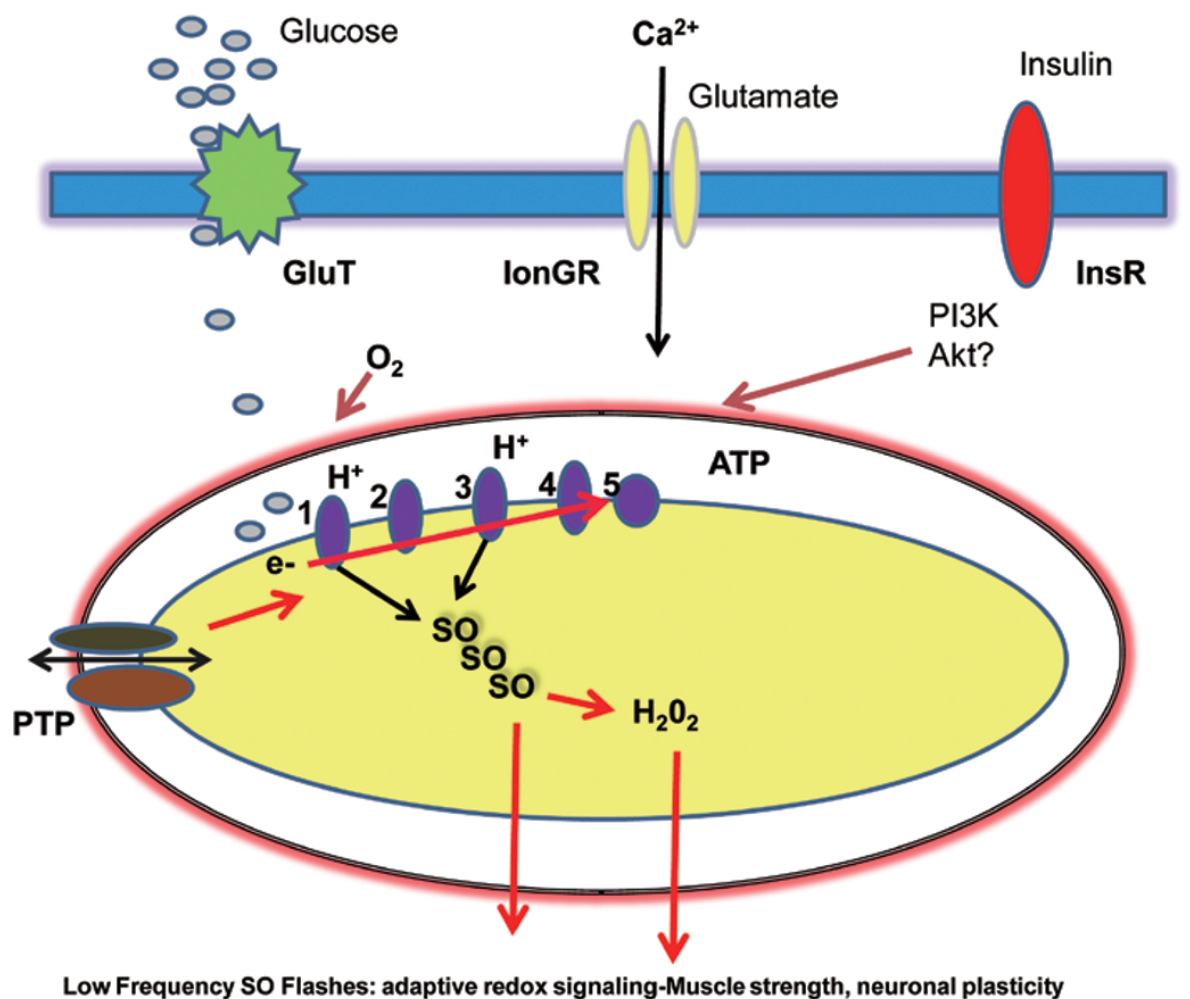

High Frequency SO Flashes: Cell dysfunction and damage-Diabetes, neurodegenerative disorders

Figure 1 Model of the mechanisms by which mitochondrial superoxide (SO) flashes are generated, and potential roles for SO flashes in physiological processes and disease in muscle and nerve cells. SO is generated primarily at complexes 1 and 3 in the electron transport chain at the inner mitochondrial membrane. Spontaneous bursts of SO (SO flashes) occur and the frequency of SO flashes can be increased by elevating the levels of glucose and $\mathrm{O}_{2}$, and in response to $\mathrm{Ca}^{2+}$ influx through plasma membrane channels such as ionotropic glutamate receptors (glutamate is the major excitatory neurotransmitter in the central nervous system). Insulin signaling can also enhance the generation of mitochondrial SO flashes. The transient opening of mitochondrial membrane permeability transition pores (PTP) is essential for SO flash production, possibly via interactions of PTP proteins with ETC proteins, or by movement of an ion or protein through the PTP. Relatively low levels of SO flashes may activate adaptive stress response signaling pathways, resulting in an increase in the expression of proteins that protect the cells against more severe stress. Prolonged high frequency SO flashes, caused by hyperoxia, high glucose levels and excessive activation of glutamate and insulin receptors, can cause oxidative damage to various cellular components resulting in dysfunction and disease, as may occur in diabetes and neurodegenerative disorders such as Alzheimer's disease. Akt, Akt kinase; ATP, adenosine triphosphate; GluT, glucose transporter; IonGR, ionotropic glutamate receptor; InsR, insulin receptor; PI3K, phosphatidylinositol-3-kinase; PTP, permeability transition pore. 1, 2, 3, 4 and 5, electron transport chain complexes 1, 2, 3, 4 and 5.

actively transported along the axons [9]. Additional experiments in the new study showed that, as was the case in cell culture, the SO flashes are associated with and require the opening of $\mathrm{MPTP}$.

Diabetes is defined by a defect in the regulation of circulating glucose levels as the result of impaired sensitivity of cells to insulin (type 2 diabetes) or failure of pancreatic $\beta$-cells to produce sufficient amounts of insulin (type 1 diabetes). There is considerable evidence that mitochondrial alterations and oxidative stress play important roles in the development of insulin resistance in muscle cells and damage to pancreatic $\beta$-cells in diabetes [4]. Because the impact of elevated blood glucose levels and insulin on the production of SO by individual mitochondria was previously unknown, Fang et al. [9] imaged mt-cpYFP fluorescence in skeletal muscle cells during the administration of glucose or insulin to the mice. There was a marked increase in the SO flash frequency of mitochondria in response to systemic administration of either glucose or insulin. The amplitude and duration of individual SO flashes were unchanged in the presence of elevated glucose and insulin levels and, interestingly, there was an apparent shift in the gating mode of the transient $\mathrm{mPTP}$ 
opening. Collectively, these new findings suggest the involvement of alterations in MPTPs and increased generation of SO flashes in the pathogenesis of diabetes, and suggest a specific role for SO flash generation in the mechanism by which muscle cells become resistant to insulin $[10,11]$.

The new findings of Fang et al. reveal several previously unknown aspects of mitochondrial dynamics in muscle cells and neurons in vivo including: (1) Both types of excitable cells generate transient quantal SO flashes; (2) The SO flashes may occur simultaneously throughout a mitochondrial reticulum (muscle cells) or independently of other mitochondria within the cell (neurons). Mitochondrial SO flashes are associated with transient mitochondrial membrane depolarization/mPTP opening; (3) The frequency of mitochondrial PTP opening/SO flashes increases in response to increased glucose availability and insulin signaling; (4) Mitochondria in the axons of neurons exhibit PTP opening/SO flashes when they are stationary and few of no PTP openings/SO flashes when they are in the process of being transported in either direction along the axons. This is a remarkable amount of new information that should be of great interest to investigators in the fields of mitochondrial biology, physiology and disease.

Many new questions arise from the data obtained using the novel mt-cpYFP probe $[8,9]$. First, what is the physiological function(s) of mitochondrial SO flashes? Their quantal nature suggests that SO flashes in mitochondria may play roles in intercellular and interorganellar signaling in a manner akin to vesicular release of neurotransmitters, endoplasmic reticulum $\mathrm{Ca}^{2+}$ sparks and coupling of $\mathrm{ER} \mathrm{Ca}^{2+}$ release and mitochondrial $\mathrm{Ca}^{2+}$ uptake $[12,13]$. The latter question leads directly to the question of what is the mechanism that determines the size of a quantum of $\mathrm{SO}$ ? Perhaps there is a pool of sequestered $\mathrm{SO}$ analogous to what has been proposed as the mechanism for release of quanta of $\mathrm{Ca}^{2+}$ through inositol triphosphate receptors in the endoplasmic reticulum [14]. Third, what is the molecular basis of the coupling of mPTP opening and SO flash generation? This could occur if opening of the PTP results in an interaction of PTP or associated proteins with proteins that are part of or associated with mitochondrial complex 1 . Alternatively, a substance (e.g., ion, peptide or protein) may move through the PTP and interact with ETC proteins. Fourth, how is the frequency of the SO flashes modified by physiological signaling and in pathological settings? Fang et al. [9] found that when mice were administered either glucose or insulin the frequency of the mitochondrial SO flashes increased. Glucose might increase SO flash frequency by providing more substrate for oxidative phosphorylation, and insulin might act by increasing glucose transport. On the other hand, activation of signaling cascades that modify activity of mitochondrial ETC activity is also a possibility for which there is precedence (Figure 1). For example, the activation of glutamate receptors in neurons has been reported to increase mitochondrial SO production by a mechanism involving $\mathrm{Ca}^{2+}$ influx through ionotropic glutamate receptors in the plasma membrane [15].

Finally, what is to be learned from the observation that SO flashes are reduced or absent in mitochondria that are being actively transported along axons [9]? Apparently, there is a mechanism by which PTP opening is inhibited when mitochondria are being actively transported along mitochondria. It has been observed that mitochondria stop moving and reside for long time periods at sites where ATP demand is particularly high including at nodes of Ranvier in myelinated axons, the base of the axon and in presynaptic terminals [16]. Kinesin(s) is the motor protein for anterograde transport and dynein(s) is the motor for retrograde transport of mitochondria in axons [16]. Mitochondria associate with the motor proteins via specific adaptor proteins including Miro, Milton and syntabulin (kinesin), and dynactin (dynein) [5]. Other proteins have been identified that function in the docking of mitochondria at specific sites within axons, with syntaphilin being one example. Perhaps one or more of these mitochondrial transport and/or docking proteins interact with proteins of the SO-generating electron transport complex or with the PTP to either inhibit or enable SO flashes. The development of mt-cpYFP and the demonstration of its utility in the visualization of SO flashes in muscle and nerve cells in vivo, combined with existing molecular genetic methods for manipulating the expression of specific proteins, suggests that it is likely that many of these questions will be answered in the not too distant future.

\section{Acknowledgments}

Supported by the Intramural Research Program of the National Institute on Aging, NIH.

\section{References}

1 Williams RJ. Cation distributions and the energy status of cells. $J$ Bioenerg 1970; 1:215-225.

2 Mattson MP. Metal-catalyzed disruption of membrane protein and lipid signaling in the pathogenesis of neurodegenerative disorders. Ann N Y Acad Sci 2004; 1012:37-50.

3 Tsutsui H, Kinugawa S, Matsushima S. Mitochondrial oxidative stress and dysfunction in myocardial remodelling. Cardiovasc Res 2009; 81:449-456.

4 Kim JA, Wei Y, Sowers JR. Role of mitochondrial dysfunction in insulin resistance. Circ Res 2008; 102:401-414.

5 Mattson MP, Gleichmann M, Cheng A. Mitochondria in neuroplasticity and neurological disorders. Neuron 2008; 60:748-766.

6 Afanas'ev IB. Signaling functions of free radicals superoxide \& nitric oxide under physiological \& pathological conditions. Mol Biotechnol 2007; 37:2-4.

7 Thomas B, Beal MF. Parkinson's dis- 
ease. Hum Mol Genet 2007; 16:R183R194.

8 Wang W, Fang H, Groom L, et al. Superoxide flashes in single mitochondria. Cell 2008; 134:279-290.

9 Fang H, Chen M, Shang W, et al. Imaging superoxide flash and metabolismcoupled mitochondrial permeability transition in living animals. Cell Res 2011; 21:1295-1304.

10 Hoehn KL, Salmon AB, HohnenBehrens $C$, et al. Insulin resistance is a cellular antioxidant defense mechanism. Proc Natl Acad Sci USA 2009; 106:17787-17792.

11 Liu HY, Cao SY, Hong T, Han J, Liu
$\mathrm{Z}$, Cao W. Insulin is a stronger inducer of insulin resistance than hyperglycemia in mice with type 1 diabetes mellitus (T1DM). J Biol Chem 2009; 284:27090-27100.

12 Hajnóczky G, Csordás G, Madesh M, Pacher P. The machinery of local $\mathrm{Ca}^{2+}$ signalling between sarco-endoplasmic reticulum and mitochondria. $J$ Physiol 2000; 529:69-81.

13 Wang SQ, Stern MD, Ríos E, Cheng H. The quantal nature of $\mathrm{Ca}^{2+}$ sparks and in situ operation of the ryanodine receptor array in cardiac cells. Proc Natl Acad Sci USA 2004; 101:3979-3984.

14 Guerrero-Hernandez A, Dagnino-
Acosta A, Verkhratsky A. An intelligent sarco-endoplasmic reticulum $\mathrm{Ca}(2+)$ store: Release and leak channels have differential access to a concealed $\mathrm{Ca}(2+)$ pool. Cell Calcium 2010; 48:143-149.

15 Yang JL, Tadokoro T, Keijzers G, Mattson MP, Bohr VA. Neurons efficiently repair glutamate-induced oxidative DNA damage by a process involving CREB-mediated up-regulation of apurinic endonuclease 1. J Biol Chem 2010; 285:28191-28199.

16 Cai Q, Sheng ZH. Mitochondrial Transport and Docking in Axons. Exp Neurol 2009; 218:257-267. 